\title{
Pulsed electron beam propagation in argon and nitrogen gas mixture
}

\author{
G. E. Kholodnaya, R. V. Sazonov, D. V. Ponomarev, G. E. Remnev and Igor Zhirkov
}

\section{Linköping University Post Print}

\section{Tweet}

N.B.: When citing this work, cite the original article.

Original Publication:

G. E. Kholodnaya, R. V. Sazonov, D. V. Ponomarev, G. E. Remnev and Igor Zhirkov, Pulsed electron beam propagation in argon and nitrogen gas mixture, 2015, Physics of Plasmas, (22), 10.

http://dx.doi.org/10.1063/1.4934608

Copyright: American Institute of Physics (AIP)

http://www.aip.org/

Postprint available at: Linköping University Electronic Press

http://urn.kb.se/resolve?urn=urn:nbn:se:liu:diva-123161 


\section{AIP | Physisos of}

\section{Pulsed electron beam propagation in argon and nitrogen gas mixture}

G. E. Kholodnaya, R. V. Sazonov, D. V. Ponomarev, G. E. Remnev, and I. S. Zhirkov

Citation: Physics of Plasmas 22, 103116 (2015); doi: 10.1063/1.4934608

View online: http://dx.doi.org/10.1063/1.4934608

View Table of Contents: http://scitation.aip.org/content/aip/journal/pop/22/10?ver=pdfcov

Published by the AIP Publishing

\section{Articles you may be interested in}

Investigation of the propagation of a gigawatt pulsed electron beam in compositions of high-pressure gas

Phys. Plasmas 21, 072302 (2014); 10.1063/1.4886152

Experiments and theory of an upstream ionization instability excited by an accelerated electron beam through a current-free double layer

Phys. Plasmas 13, 122101 (2006); 10.1063/1.2398929

On intense electron beam propagation through gas jets

Phys. Plasmas 13, 033101 (2006); 10.1063/1.2176600

Control of plasma parameters by using noble gas admixtures

J. Vac. Sci. Technol. A 22, 2131 (2004); 10.1116/1.1772375

Precise control of atomic nitrogen production in an electron cyclotron resonance plasma using $\mathrm{N} 2$ /noble gas mixtures

Appl. Phys. Lett. 73, 456 (1998); 10.1063/1.121898

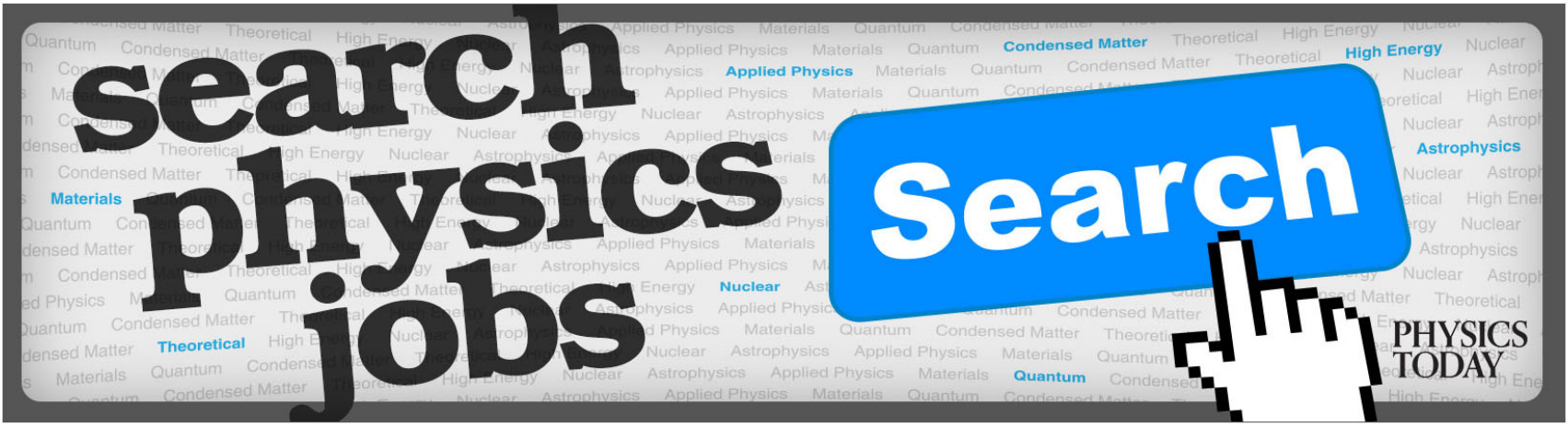




\title{
Pulsed electron beam propagation in argon and nitrogen gas mixture
}

\author{
G. E. Kholodnaya, ${ }^{1}$ R. V. Sazonov, ${ }^{1}$ D. V. Ponomarev, ${ }^{1}$ G. E. Remnev, ${ }^{1}$ and I. S. Zhirkov ${ }^{2}$ \\ ${ }^{1}$ High Technology Physics Institute, Tomsk Polytechnic University, 2 a Lenin Avenue, 634028 Tomsk, Russia \\ ${ }^{2}$ Department of Physics, Chemistry and Biology (IFM), Linköping University, SE-581 83 Linköping, Sweden
}

(Received 4 August 2015; accepted 13 October 2015; published online 27 October 2015)

\begin{abstract}
The paper presents the results of current measurements for the electron beam, propagating inside a drift tube filled in with a gas mixture $\left(\mathrm{Ar}\right.$ and $\left.\mathrm{N}_{2}\right)$. The experiments were performed using the TEA-500 pulsed electron accelerator. The main characteristics of electron beam were as follows: $60 \mathrm{~ns}$ pulse duration, up to $200 \mathrm{~J}$ energy, and $5 \mathrm{~cm}$ diameter. The electron beam propagated inside the drift tube assembled of three sections. Gas pressures inside the drift tube were $760 \pm 3$, $300 \pm 3$, and $50 \pm 1$ Torr. The studies were performed in argon, nitrogen, and their mixtures of $33 \%, 50 \%$, and 66\% volume concentrations, respectively. C 2015 AIP Publishing LLC.

[http://dx.doi.org/10.1063/1.4934608]
\end{abstract}

\section{INTRODUCTION}

Studies of the electron beam propagation in gases are of interest not only for the development of physical representations about the multiple scattering phenomenon and the formation of charged and excited particles but also are very promising for application purposes. For example, they are employed in the pulsed plasma chemical technology, which is applied to synthesize the nanosized metal oxides, ${ }^{1-6}$ and in the pulsed technology, which allows industrial and domestic wastewater treatment and disinfection, ${ }^{7,8}$ etc. ${ }^{9,10}$

Nowadays, there are many experimental and theoretical works studying the electron beam propagation in different gases $^{11-20}$ and the main characteristics of an electron beam behaviour during its propagation. In this work, we report about how to increase the efficiency of beam energy transfer varying the configuration of drift tubes. When an electron beam is injected into a gas medium, due to the final timing of gas ionization, the beam leading edge loses particles at the drift tube walls as a result of an action of radial electric fields of the space charge. However, on their way, the electrons manage to ionize the gas, and the remaining part of the beam becomes charge-compensated (the charge neutralization process $^{11-15}$ ). Depending on the ratio of electrons $n_{e}$ and ions $n_{i}$ in the beam volume unit, it reaches a partial compensation $\left(\mathrm{n}_{\mathrm{e}}>\mathrm{n}_{\mathrm{i}}\right)$, a total compensation $\left(\mathrm{n}_{\mathrm{e}}=\mathrm{n}_{\mathrm{i}}\right)$, or becomes even overcompensated, when $\mathrm{n}_{\mathrm{e}}<\mathrm{n}_{\mathrm{i}}{ }^{13-15}$ The authors present the results of experiments, in which the electron beam was focused by the ion background arising under conditions of the electron beam injection into argon (0.06-0.2 mTorr pressure range). ${ }^{21}$ The electron energies were of $1.5 \mathrm{MeV}-2 \mathrm{MeV}$.

In addition to the charge compensation, it is necessary to consider the process associated with the current neutralization. $^{11-19}$

In the course of electron transportation, the force, directed opposite to repulsive forces, arises. This force is caused by a magnetic field generated by the electrons moving in the opposite direction. The magnetic field acts on the beam so that it tends to compress, preventing its defocusing.

Investigation results for an electron beam propagating in vacuum and in gases (nitrogen, helium, and carbon dioxide) have been presented. ${ }^{22}$ The experiments were performed using the accelerator with the following parameters: $430 \mathrm{keV}$ electron energy, $10 \mathrm{kA}$ beam current, and $15 \mathrm{~ns}$ current pulse duration. The beam current was registered using the Faraday cup, located at a fixed distance inside the drift tube. The authors $^{22}$ registered an insufficient gas ionization in the case of electron beam propagation in nitrogen under 50 mTorr pressure. In this case, beam charge compensation was also insufficient. The electron beam dissipated under an action of electric repulsive forces. However, the current registered using the Faraday cup was much higher in nitrogen than in vacuum for the same distances. When the pressure inside the drift tube reached 100 mTorr, the effective ionization occurred, and the volume charge neutralization realized. Under increased pressures inside the drift tube, the leading role of current compensation in the process of electron beam propagation, for all types of gases used within these experiments, became evident. ${ }^{22}$ The authors pointed out that their experimental results agreed well with the Lawson's predictions. ${ }^{11,12}$ The authors have presented the results of the experimental and theoretical studies for pulsed electron beams propagating inside drift tubes filled with $\mathrm{N}_{2}$ under pressures ranging from $10^{-3}$ to $\sim 10^{3}$ Torr. $^{23}$ The experiments were performed using the Hermes-III accelerator with the following parameters: $19 \mathrm{MeV}$ electron energy, $700 \mathrm{kA}$ beam current, and $25 \mathrm{~ns}$ duration. The obtained results showed that for the given type of electron beams, there were two ranges of efficient propagation, which were associated with nitrogen pressure. The first range is from 1 to $100 \mathrm{mTorr}$ (a lowpressure area) and the second one is from 1 to 100 Torr (a high-pressure area). Beyond these pressure ranges, the electron beam was developing the instability, which, finally, resulted in its full breakdown. The beam propagating under the first range became significantly ionized, and, the volume ion charge, which was formed after plasma ion generation, restricted it. The paper has reported about high-energy DC electron beams of about $100 \mathrm{keV}$ energy propagating in a rarefied gas. ${ }^{24}$ The authors studied the propagation of partially and totally charge-neutralized electron beams, the ranges of gas pressures, and other parameters enabling the efficient energy transfer in gas media. The electron beam of 
$60 \mathrm{keV}$ energy and $270 \mathrm{~mA}$ current was injected into nitrogen under $10^{-6}$ to $10^{-5}$ Torr pressures. Models, describing the radial neutralization distributions in two different cases of incomplete and complete neutralization, have been developed. The experimental data and the calculations were in good agreement. The obtained results confirmed that under complete charge neutralization, the dependence of beam current density distribution was linear, and under partial charge neutralization, the beam current density distribution was nonlinear. The pulsed electron beam was propagating in a low-pressure gas. ${ }^{25}$ The authors used a drift tube with an external magnetic field. As a result, the electron beam had become self-pinched at about $10 \mathrm{~Pa}$ pressures of nitrogen, air, etc.

Analysing the above-mentioned works, we can conclude that, increased gas pressures changed the electric to magnetic field force ratio, revolutionizing, in this way, a behaviour of the beam propagating inside a drift tube. When pressures inside the drift chamber were below $10^{-3}$ Torr, the radial repulsive forces exceed all others, causing a beam dissipation. If the chamber was filled with a gas of about $10^{-2}$ Torr pressure, the beam propagation mechanism crucially changed. The electrons ionized the gas, releasing secondary electrons, which were repulsed in radial directions outwards of the beam. The remaining positive ions neutralized the beam charge, diminishing the repulsion, while the forces of magnetic compression reduced its diameter. If the pressure subsequently increased up to $\mathrm{p}=10^{-1}$ Torr, the gas density was enough to form a well-conducting plasma. The DC induction field caused a reverse current in this plasma, which reinforced a focusing action of magnetic field on the beam. An electron flow was freely drifting with a very low divergence, since all the forces of the beam were balanced. At this pressure, the flow intensity significantly decreased with distance due to the gas molecule repulsion. The electron beam propagation in the gas medium was accompanied by a deceleration and dissipation of beam particles, a gas heating, and an ionization with formation of plasma channels. It also depended on the beam parameters (the electron energy, the current), gas pressure, and sort. The deceleration and dissipation of beam electrons arise from the Coulomb interactions with electrons and ions of gas atoms. The authors studied another important physical process, which influenced the beam propagation in gases. ${ }^{26-28}$ This process was associated with various types of developing instabilities. These instabilities were the main reason of degradation of transportation characteristics of the electron beams propagating in gas media. The instabilities significantly deformed an equilibrium configuration of the beam. One of the most frequent type of instabilities was the firehose resistive instability, the development of which shifted the entire beam, ejecting it onto the walls of the drift tube.

The literature analysis revealed numerous researches dealing with the physics of electron beams propagating in low-pressure gases. However, the studies concerning the interaction of pulsed electron beams with high-pressure gas mixtures were not numerous. ${ }^{29-31}$ The aim of the present paper was the experimental study of pulsed electron beam propagation in argon and nitrogen mixtures. The choice of gas compositions was explained by their wide application in technological processes of the most industrial technologies. ${ }^{32,33}$

\section{EXPERIMENTAL SETUP}

The electron beam propagation in the gas mixture (argon and nitrogen) has been studied using the TEA-500 accelerator. ${ }^{34,35}$ The basic parameters of accelerator were as follows: $500 \mathrm{keV}$ accelerating voltage, $10 \mathrm{kA}$ current electron beam, $60 \mathrm{~ns}$ half-amplitude pulse duration, up to $200 \mathrm{~J}$ pulse energy, and up to 5 pps pulse repetition rate. The electron beam was formed by explosion emission of a graphite cathode of $45 \mathrm{~mm}$ diameter. Then, the beam was extracted through the anode window, which was assembled of a support grid (with $95 \%$ optical transparency) and a $140-\mu \mathrm{m}$ thick aluminium foil. The anode-cathode gap for all the experiments was $13 \mathrm{~mm}$. The stability of accelerator operation was controlled using the Rogowski coil and capacitor voltage divider (Fig. 1, the current and the voltage oscillogram). The charge of extracted electrons was $390-430 \mu \mathrm{C}$. The sensorregistered variation of accelerating voltage and the registered beam current did not exceed $5 \%$.

The pulsed electron beam was injected inside a $40-\mathrm{cm}$ long metal drift tube. The drift tube was equipped with three reverse current shunts. The sketch of experimental stand is presented in Fig. 2.

The experiments were performed as follows: the drift tube was filled in with a gas medium under study and the electron beam was injected inside, the current oscillograms were simultaneously recorded from three reverse current shunts. First, the experiments were performed in argon under 760, 300, and 50 Torr pressures, then in nitrogen under the same pressures. Thereafter, the electron beam was injected in argon-nitrogen mixtures of various volume concentrations. Pressures inside the drift tube were 760,300 , or 50 Torr, and the ratios were Ar: $\mathrm{N}_{2}=1: 1, \operatorname{Ar}: \mathrm{N}_{2}=1: 2$, and Ar: $\mathrm{N}_{2}=2: 1$.

\section{RESULTS AND DISCUSSION}

The results of experimental researches of the pulsed electron beam propagation in argon and nitrogen are

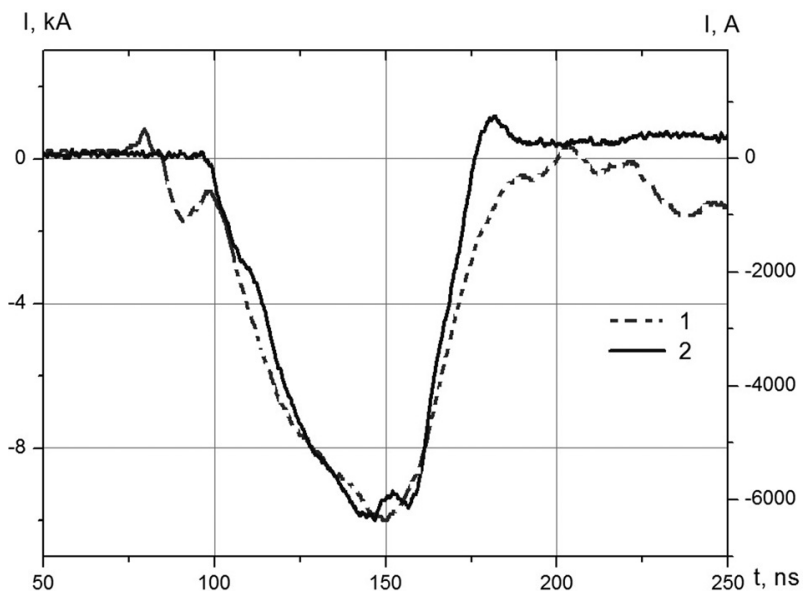

FIG. 1. Oscillograms of the current fed to the diode assembly (1) and of the injected into the drift tube beam current (2). Oscillograms were averaged to five successive pulses. 


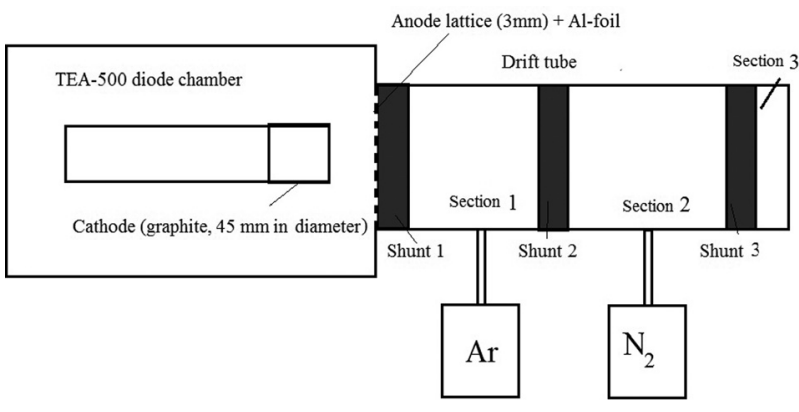

FIG. 2. Scheme of the experimental stand to study pulsed electron beam propagation in gases using reverse current shunts.

presented in Figs. 3-5. The figure shows the oscillograms of the accelerating voltage (1), the electron current closing on the walls of the first section of drift tube (2), the electron current closed on the walls of the second section of drift tube (3), the electron current reaching the back flange of drift tube-Section III (4), and the total reverse current closed on the walls of drift tube (5).

When the pulsed electron beam propagated in argon under 50,300, and 760 Torr pressures, the back edge of signals coming from the electron current closed on the walls of the first and the second sections of drift tube, of those coming from the electron current having reached the back flange of drift tube, and of total reverse current closing on the walls of drift tube tailed. This tailing indicated that in Ar, the lifetime of the plasma arising in the course of pulsed electron beam travelling was longer than in $\mathrm{N}_{2}$. The reverse current shunt signals tended to tail in the experiments with an increased Ar fraction of the gas mixture $\left(\mathrm{Ar}+\mathrm{N}_{2}\right)$. For argon, the amplitudes of the current electrons closing on the first and the second sections of drift tube were the same within the limits of experimental error for all pressures under study $(50,300$, and 760 Torr). When the pressure inside the drift tube was 50 Torr, the current amplitude of electrons, which reached the back flange of drift tube, was $2 \mathrm{kA}$, while under 300 and 760 Torr pressures, its value did not exceed $1 \mathrm{kA}$. The pulsed electron beam dissipation on the drift tube walls was maximum in the range of the given pressures.

The main feature of the experiments on the electron beam propagation in argon is that the amplitude of entire

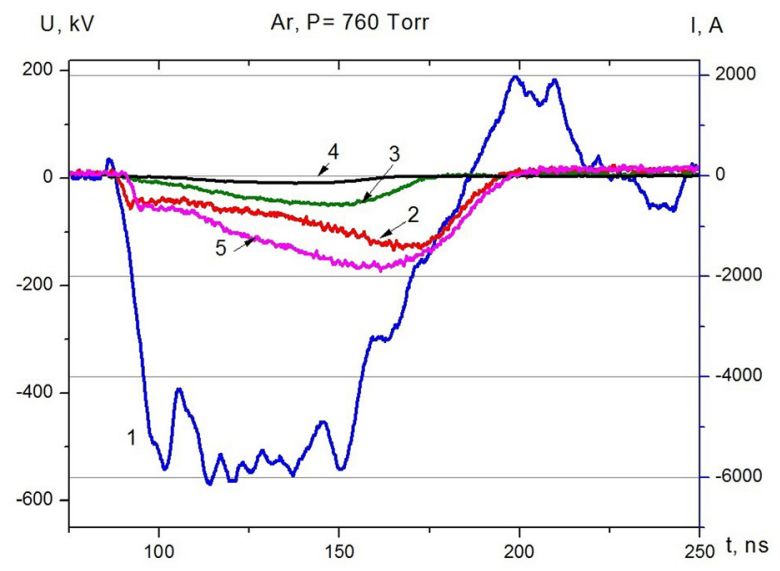

reverse current closing on the drift tube walls was lower in comparison with that of injected beam current $(6 \mathrm{kA})$ (Fig. 1). The amplitude of the entire reverse current was $3.2 \mathrm{kA}$ at 50 Torr pressure, and $2 \mathrm{kA}$ at 300 and 760 Torr. We assume that the difference between injected and measured number of electrons was due to the ionization of Ar molecules and the subsequent Ar recombination (a significant number of electrons was absorbed by the medium). The participation of drift tube structural elements in recombination was minimum. The formation of a virtual cathode preventing the beam propagation inside the drift tube and the subsequent beam current closing along the plasma skin-layer were also probable.

We have performed a calorimetric study of the pulsed electron beam propagation in argon under 50,300, and 760 Torr pressures, which verified that the virtual cathode was not formed. ${ }^{36}$ This allowed us to conclude that the propagating in argon pulsed electron beam almost avoided dissipation on the drift tube walls. The plasma conductivity was sufficient, and the beam closed along it. Under pulsed electron beam propagation in nitrogen, the value of the total reverse current amplitude closing on the drift tube walls for 50,300 , and 760 Torr pressures was the same (about $4.2 \mathrm{kA}$ ) within the error limits. This value was also lower than that of the beam current injected into the drift tube. In nitrogen, the plasma was formed in the same way as in argon, and the remaining part of the electrons were closing along. In nitrogen, under 760 Torr pressure, the electron current amplitude coming from the back flange of drift tube was $0.9 \mathrm{kA}$. The dissipation of the propagating in nitrogen electron beam on the drift tube walls was more substantial in comparison with argon under 760 Torr pressure. For other pressures of argon and nitrogen, the values of current amplitudes registered for the electrons closing along the first and the second sections of drift tube were the same within the limits of experimental error.

Fig. 6 presents the results obtained for the nitrogenargon composition. In this case, we employed the values of charges falling on the walls and reaching the back flange of drift tube.

Analysing the results of studies obtained for the pulsed electron beam propagating in the gas compositions under

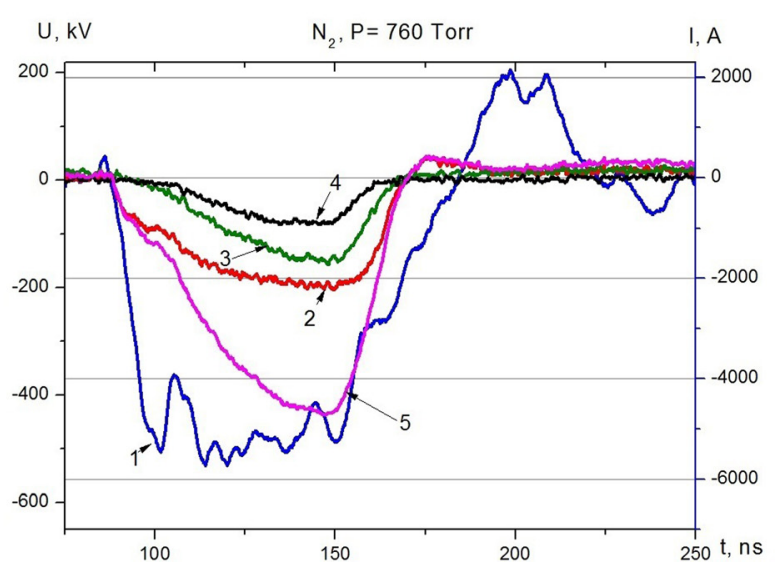

FIG. 3. Values of the accelerating voltage and the beam currents registered by shunts (three successive pulses), 760 Torr pressure inside the drift tube. 

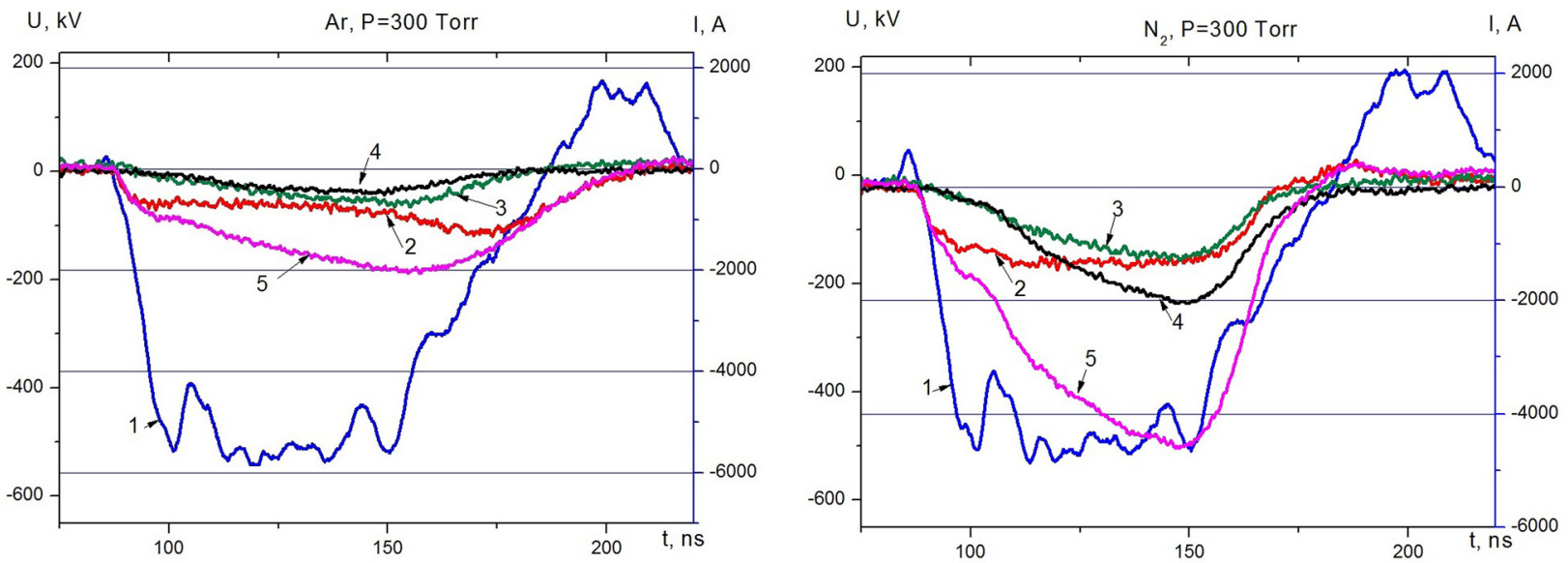

FIG. 4. Values of the accelerating voltage and the beam currents registered by shunts (three successive pulses), 300 Torr pressure inside the drift tube.
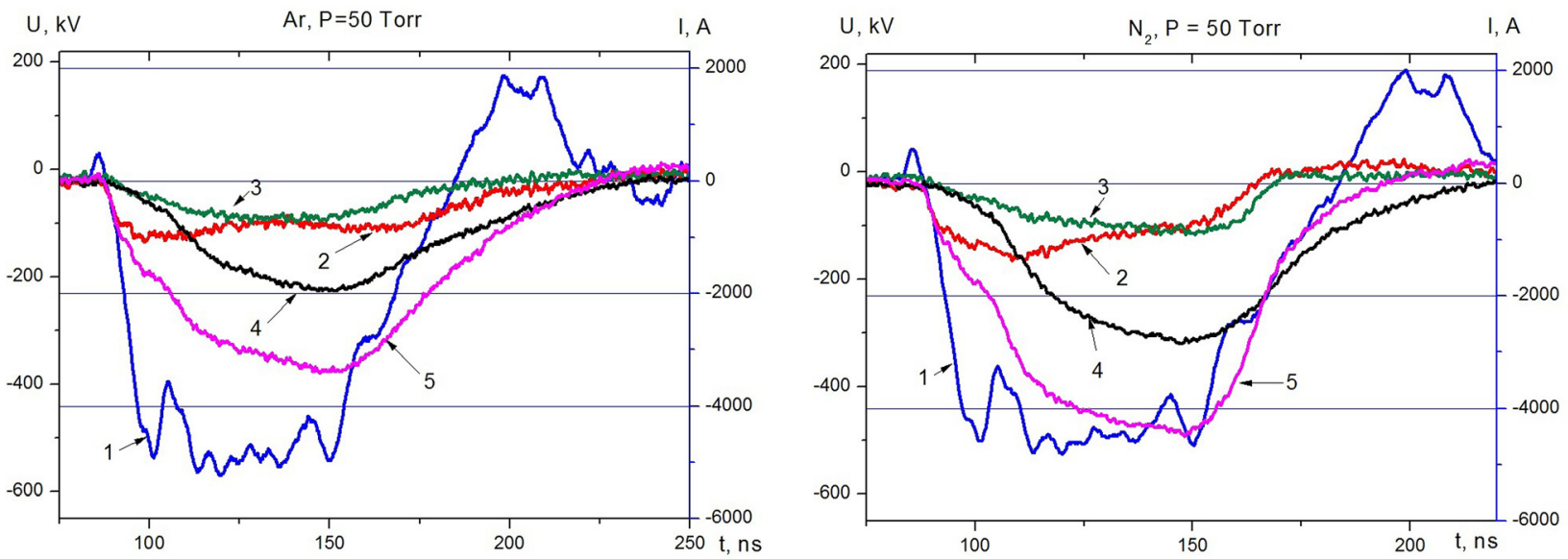

FIG. 5. Values of the accelerating voltage and beam currents registered by shunts (three successive pulses), 50 Torr pressure inside the drift tube.
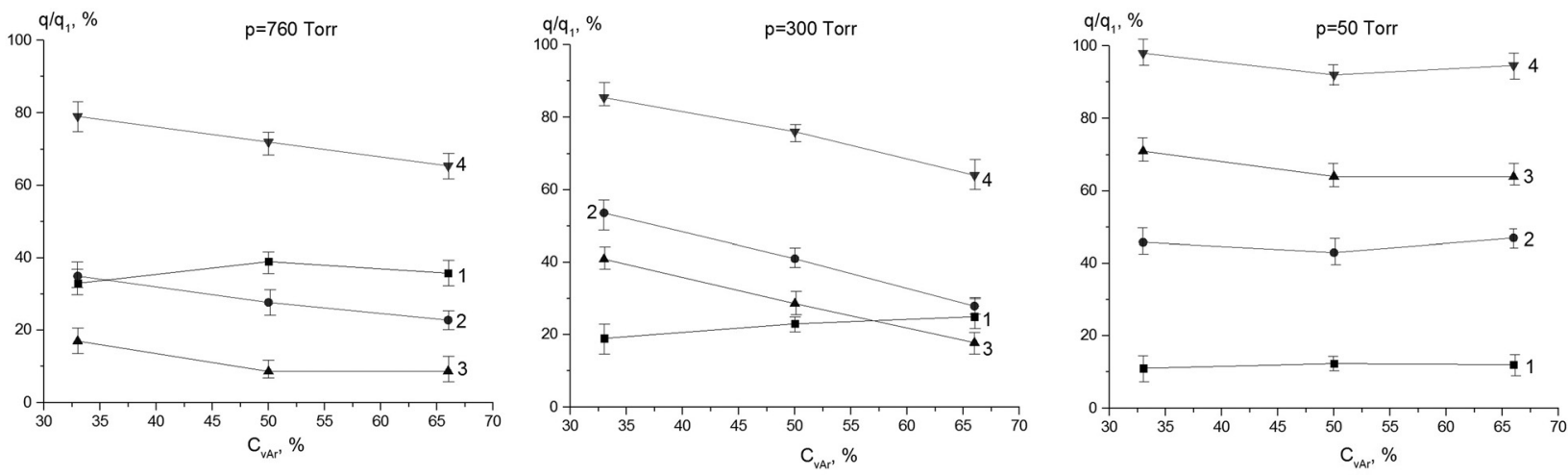

FIG. 6. The dependence of the value ratios of electron charge (q) falling on the walls of the first section of drift tube (1), falling on the walls of the second section of drift tube (2), reaching the back flange of drift tube (3), and closing on the walls of drift tube (4) to the charge $\left(\mathrm{q}_{1}\right)$ injected into the drift tube on the argon volume concentration for 50,300 , and 760 Torr pressures.

study, the entire pressure inside the drift tube 760 Torr, and $\mathrm{N}_{2}: \mathrm{Ar}=2: 1$ ratio, we have seen that the value ratios of electron charge falling on the walls of the first and second sections of drift tube were the same within the limits of experimental error. When the volume concentration of argon inside the drift tube increased, the ratio of all $\mathrm{q} / \mathrm{q}_{1}$ values decreased. In the case, when the total pressure inside the drift tube was 50Torr, for $\mathrm{N}_{2}: \mathrm{Ar}=2: 1, \quad \mathrm{~N}_{2}: \mathrm{Ar}=1: 1$, and $\mathrm{N}_{2}: \operatorname{Ar}=1: 2$, the value ratio of electron charge $(\mathrm{q})$ falling on the walls of the first section of drift tube and of those $\left(q_{1}\right)$ injected into the drift tube was similar within the limits of experimental error. The value ratio of electron charge falling on the walls of the second section of drift tube, reaching the back flange of drift tube, and closing all over the walls of drift tube was similar under 50 Torr pressure. Under 300 Torr and increased argon volume concentration, the ratio of the value of the electron charge falling on the walls of the first section of drift tube to that of the electron charge injected 
into the drift tube increased, while the value of the charge of electrons falling on the walls of drift tube decreased. In this case, the pulsed electron beam dissipated on the walls of the first section of drift tube. It seemed to be associated with recombination and relaxation processes occurring in plasmas of various compositions.

\section{CONCLUSION}

The work has presented the research results on the pulsed electron beam propagating in argon, nitrogen, and a gas mixture (argon and nitrogen) under 760, 300, and 50 Torr pressures. In nitrogen, the pulsed electron beam propagated more efficiently in comparison with argon, under all pressures studied within the present work. Under 50 Torr pressure, a change of argon concentration in nitrogen of $33 \%-60 \%$ did not have any effect on the pulsed electron beam dissipation. Under 300 and 760 Torr pressure, an addition of argon to nitrogen of 33\%-66\% concentration decreased the electron beam dissipation in the initial region of drift tube. The maximum absorption of the electron beam occurred in argon. Gas compositions of high argon volume concentrations require the highest beam energy embedding. This allows us to conclude that argon is very promising as an efficient buffer gas for different technological processes.

\section{ACKNOWLEDGMENTS}

The research was carried out with the financial support of State Task "Science," Ministry of Education and Science of the Russian Federation (Project No.: 11.939.2014/k).

${ }^{1}$ S. Yu. Sokovnin and V. G. Il'Ves, "Production of nanopowders using pulsed electron beam," Ferroelectrics 436(1), 101-107 (2012).

${ }^{2}$ M. E. Balezin, V. V. Bazarnyi, E. A. Karbovnichaya, and S. Y. Sokovnin, "Application of nanosecond electron beam for production of silver nanopowders," Nanotechnol. Russia 6(11-12), 757-762 (2011).

${ }^{3}$ V. G. Il'Ves and S. Y. Sokovnin, "Production of $\mathrm{ZnO}$ and $\mathrm{Zn}-\mathrm{ZnO}$ nanopowders using evaporation by a pulsed electron beam in low-pressure gas," Nanotechnol. Russia 6(1-2), 137-143 (2011).

${ }^{4}$ D. V. Ponomarev, G. E. Remnev, R. V. Sazonov, and G. E. Kholodnaya, "Pulse plasma-chemical synthesis of ultradispersed powders of titanium and silicon oxide," IEEE Trans. Plasma Sci. 41(10), 2908-2912 (2013).

${ }^{5}$ R. Sazonov, G. Kholodnaya, D. Ponomarev, G. Remnev, and O. Razumeko, "Plasma-chemical synthesis of composite nanodispersed oxides," J. Korean Phys. Soc. 59(6), 3508-3512 (2011).

${ }^{6}$ G. Kholodnaya, D. Ponomarev, R. Sazonov, and G. Remnev, "Characteristics of pulsed plasma-chemical synthesis of silicon dioxide nanoparticles," Radiat. Phys. Chem. 103, 114-118 (2014).

${ }^{7}$ W. A. Parejo Calvo, C. L. Duarte, L. D. Machado, J. E. Manzoli, A. B. Geraldo, Y. Kodama, L. G. Silva, E. S. Pino, E. S. R. Somessari, C. G. Silveira, and P. R. Rela, "Electron beam accelerators-trends in radiation processing technology for industrial and environmental applications in Latin America and the Caribbean," Radiat. Phys. Chem. 81(8), 1276-1281 (2012).

${ }^{8}$ S. Dånmark, A. Finne-Wistrand, K. Schander, M. Hakkarainen, K. Arvidson, K. Mustafa, and A. C. Albertsson, "In vitro and in vivo degradation profile of aliphatic polyesters subjected to electron beam sterilization," Acta Biomater. 7(5), 2035-2046 (2011).

${ }^{9}$ P. Guerra, J. Udías, E. Herranz, J. Santos-Miranda, J. Herraiz, M. Valdivieso, R. Rodríguez, J. Calama, J. Pascau, F. Calvo, C. Illana, M. Ledesma-Carbayo, and A. Santos, "Feasibility assessment of the interactive use of a Monte Carlo algorithm in treatment planning for intraoperative electron radiation therapy," Phys. Med. Biol. 59(23), 7159-7179 (2014).
${ }^{10}$ E. Hemsing, G. Stupakov, D. Xiang, and A. Zholents, "Beam by design: Laser manipulation of electrons in modern accelerators," Rev. Mod. Phys. 86(3), 897-941 (2014).

${ }^{11}$ W. H. Bennet, "Magnetically self-focusing streams," Phys. Rev. 45, 890-895 (1934).

${ }^{12} \mathrm{~N}$. Alfven, "On the motion of cosmic rays in interstellar space," Phys. Rev. 55, 425-430 (1939).

${ }^{13}$ T. X. Zhang, "Anisotropic model for resonant heating of ions by Alfvén waves,” J. Plasma Phys. 79(5), 963-971 (2013).

${ }^{14}$ G. Wallis, K. Sauer, D. Sünder, S. Rosinskii, A. Rukhadze, and V. Rukhlin, "Injection of high-current relativistic electron beams into plasma and gas," Sov. Phys. Usp. 17, 492-506 (1975).

${ }^{15}$ M. Yousfi, J. Leger, J. F. Loiseau, B. Held, O. Eichwald, B. Defoort, and J. M. Dupillier, "Electron beam transport in heterogeneous slab media from $\mathrm{MeV}$ down to eV," Radiat. Protect. Dosimeters 122(1-4), 46-52 (2006).

${ }^{16}$ D. A. Erwin and J. A. Kunc, "Transport of low- and medium-energy electron and ion beams in seawater and its vapors," Phys. Rev. A. 38, 4135 (1988).

${ }^{17}$ A. S. Artamonov, V. A. Gorbunov, N. K. Kuksanov, and R. A. Salimov, "Propagation of a steady electron beam in air," J. Appl. Mech. Tech. Phys. 22(1), 12-15 (1981).

${ }^{18}$ P. A. Miller, J. B. Gerardo, and J. W. Poukey, "Relativistic electron beam propagation in low pressure gases," J. Appl. Phys. 43(7), 3001-3007 (1972).

${ }^{19}$ M. V. Gladyshev and M. G. Nikulin, "Beam-plasma discharge in the propagation of a long-pulse relativistic electron beam in a medium-pressure rarefied gas," J. Tech. Phys. 42(5), 542-546 (1997).

${ }^{20}$ R. V. Sazonov, G. E. Kholodnaya, D. V. Ponomarev, and G. E. Remnev, "Investigation of the propagation of a gigawatt pulsed electron beam in compositions of high-pressure gas," J. Phys. Plasmas. 21(7), 072302 (2014).

${ }^{21}$ S. L. Shope, W. K. Tucker, D. E. Hasti, J. W. Poukey, G. W. Kamin, and W. W. Rienstra, "Acceleration and bending of a relativistic electron beam on the sandia recirculating linac," in Proceedings of the 1987 IEEE Particle Accelerator Conference: Accelerator Engineering and Technology; Washington, DC, USA, Code 11419, 1987.

${ }^{22}$ A. Luches, V. Nassisi, and A. Perrone, "Structures of an electron beam drifting in vacuum and in low-pressure gases," J. Appl. Phys. 50(4), 2502-2505 (1979).

${ }^{23}$ T. W. L. Sanford, "High-power electron-beam transport in long gas cells from $10^{-3}$ to $10^{3}$ Torr nitrogen," J. Phys. Plasmas. 2(6), 2539-2546 (1995).

${ }^{24}$ R. E. Rand, M. C. Lampel, and D. Y. Wang, "Transport of self-field dominated dc low-energy electron beams through low-pressure gases," J. Appl. Phys. 62(5), 1639-1654 (1987).

${ }^{25} \mathrm{X}$. L. Jiang and S. C. Jiang, "Propagation of intense pulsed electron beams with energies below $80 \mathrm{keV}$," IEEE Trans. Nucl. Sci. 32(5), 2492-2494 (1985).

${ }^{26}$ D. S. Prono, "Propagation studies using modulated relativistic electron beams." Phys. Fluids. 17(9), 1727-1737 (1974).

${ }^{27}$ A. S. Manuilov, "Effect of the return plasma current radial profile on the dynamics of resistive hose instability of a relativistic electron beam propagating in a dense gas-plasma medium," Tech. Phys. 58(1), 76-79 (2013).

${ }^{28}$ C. Ekdahl, E. O. Abeyta, P. Aragon, R. Archuleta, R. Bartsch, H. Bender, R. Briggs, W. Broste, C. Carlson, K. C. D. Chan, D. Dalmas, S. Eversole, D. Frayer, R. Gallegos, J. Harrison, T. Hughes, E. Jacquez, D. Johnson, J. Johnson, B. T. McCuistian, N. Montoya, C. Mostrom, S. Nath, D. Oro, L. Rowton, M. Sanchez, R. Scarpetti, M. Schauer, M. Schulze, Y. Tang, A. Tipton, and C. Y. Tom, "Long-pulse beam stability experiments on the DARHT-II linear induction accelerator,” IEEE Trans. Plasma Sci. 34(2), 460-466 (2006).

${ }^{29}$ M. Facina, B. Bruyneel, S. Dean, J. Gentens, M. Huyse, Y. Kudryavtsev, P. Van Den Bergh, and P. Van Duppen, "A gas cell for thermalizing, storing and transporting radioactive ions and atoms. Part II: On-line studies with a laser ion source," Nucl. Instrum. Methods Phys. Res. B 226(3), 401-418 (2004).

${ }^{30}$ J. Ishikawa, Y. Takeiri, and T. Takagi, "Axial magnetic field extractiontype microwave ion source with a permanent magnet," Rev. Sci. Instrum. 55(4), 449-456 (1984).

${ }^{31}$ J. R. Smith, W. O. Doggett, and C. M. Armstrong, "Collective ion acceleration and relativistic electron-beam propagation," J. Appl. Phys. 57(5), 1632-1637 (1985).

${ }^{32}$ R. Tumlos, J. Ting, E. Osorio, L. Rosario, H. Ramos, A. Ulano, H. Lee, and G. Regalado, "Results of the study of chemical-, vacuum drying- and 
plasma-pretreatment of coconut (Cocos nucifera) lumber sawdust for the adsorption of methyl red in water solution," Surf. Coat. Technol. 205(2), S425-S429 (2011).

${ }^{33} \mathrm{~J}$. W. Bradley and T. Welzel, "Physics and phenomena in pulsed magnetrons: An overview," J. Phys. D: Appl. Phys. 42(9), 093001 (2009).

${ }^{34}$ G. E. Remnev, E. G. Furman, A. I. Pushkarev, S. B. Karpuzov, N. A. Kondrat'ev, and D. V. Goncharov, "A high-current pulsed accelerator with a matching transformer," Instrum. Exp. Tech. 47(3), 394-398 (2004).

${ }^{35}$ Y. I. Isakova, A. I. Pushkarev, and G. E. Kholodnaya, "A differential highvoltage divider," Instrum. Exp. Tech. 54(2), 183-186 (2011).

${ }^{36}$ G. Kholodnaya, R. Sazonov, D. Ponomarev, and G. Remnev, "Propagation of the pulsed electron beam of nanosecond duration in gas composition of high pressure," J. Phys: Conf. Ser. (in press). 